\title{
BMJ Open Creation of a database to assess effects of omega-3, omega- 6 and total polyunsaturated fats on health: methodology for a set of systematic reviews
}

\author{
Lee Hooper, ${ }^{\circledR}$ Asmaa Abdelhamid, ${ }^{1}$ Julii Brainard, ${ }^{\oplus 1}$ Katherine H O Deane, ${ }^{\bullet 2}$ \\ Fujian Song, ${ }^{1}$ on behalf of the PUFAH group
}

To cite: Hooper L, Abdelhamid A, Brainard J, et al. Creation of a database to assess effects of omega- 3 , omega- 6 and total polyunsaturated fats on health: methodology for a set of systematic reviews. BMJ Open 2019;9:e029554. doi:10.1136/ bmjopen-2019-029554

- Prepublication history and additional material for this paper are available online. To view these files, please visit the journal online (http://dx.doi org/10.1136/bmjopen-2019029554 )

Received 6 February 2019 Revised 21 February 2019 Accepted 22 February 2019

\section{Check for updates}

(c) Author(s) (or their employer(s)) 2019. Re-use permitted under CC BY-NC. No commercial re-use. See rights and permissions. Published by BMJ.

${ }^{1}$ Norwich Medical School, University of East Anglia, Norwich, UK

${ }^{2}$ School of Health Sciences, University of East Anglia, Norwich, UK

Correspondence to

Dr Lee Hooper;

I.hooper@uea.ac.uk

\section{ABSTRACT}

Objective To create a database of long-term randomised controlled trials (RCTs) comparing higher with lower omega-3, omega-6 or total polyunsaturated fatty acid (PUFA), regardless of reported outcomes, and to develop methods to assess effects of increasing omega-6, alphalinolenic acid (ALA), long-chain omega-3 (LCn3) and total PUFA on health outcomes.

Design Systematic review search, methodology and meta-analyses.

Data sources Medline, Embase, CENTRAL, WHO International Clinical Trials Registry Platform, Clinicaltrials. gov and trials in relevant systematic reviews.

Eligibility criteria RCTs of $\geq 24$ weeks' duration assessing effects of increasing ALA, LCn3, omega-6 or total PUFAs, regardless of outcomes reported.

Data synthesis Methods included random-effects metaanalyses and sensitivity analyses. Funnel plots were examined, and subgrouping assessed effects of intervention type, replacement, baseline diabetes risk and use of diabetic medications, trial duration and dose. Quality of evidence was assessed using Grading of Recommendations Assessment, Development and Evaluation (GRADE).

Results Electronic searches generated 37810 hits, deduplicated to 19772 titles and abstracts. We assessed 2155 full-text papers, conference abstracts and trials registry entries independently in duplicate. Included studies were grouped into 363 RCTs comparing higher with lower omega-3, omega- 6 and/or total PUFA intake of at least 6 months' duration-the Database. Of these 363 included RCTs, 216 RCTs were included in at least one of our reviews of health outcomes, data extracted and risk of bias assessed in duplicate. Ninety five RCTs were included in the Database but not included in our current reviews. Of these 311 completed trials, 27 altered ALA intake, 221 altered LCn3 intake and 16 trials altered omega-3 intake without specifying whether ALA or LCn3. Forty one trials altered omega- 6 and 59 total PUFA.The remaining 52 trials are ongoing though $13(25 \%)$ appear to be outstanding, or constitute missing data.

Conclusions This extensive database of trials is available to allow assessment of further health outcomes.

\section{BACKGROUND}

When the WHO Nutrition Guidance Expert Advisory Group (NUGAG) Subgroup on Diet
Strengths and limitations of this study

- Extensive search for long-term trials of omega-3, omega- 6 and total polyunsaturated fatty acid.

- Search date April 2017, updated in December 2018 with rechecking of all ongoing trials.

- Provision of a database of all relevant randomised controlled trials with references to relevant papers, abstracts, theses and trials registry entries.

- Detailed methodology for the associated set of systematic reviews.

- We contacted many authors for further details of methodology and outcome data, but we were unable to contact all authors due to limited resources.

and Health commissioned us to assess the effects of omega-3, omega- 6 and total polyunsaturated fatty acids (PUFAs) across a wide range of outcomes, we realised the logical way to progress was not to carry out the 27 reviews individually. Instead, we could create a single database of all long-term randomised controlled trials (RCTs) that compared higher with lower omega-3 or omega- 6 or total PUFA regardless of outcomes reported. We could then extract all outcomes relevant to our reviews from this set of studies, from published papers, abstracts and trials registry entries. Even better, we could contact authors and ask whether they had collected any useful outcomes (perhaps as adverse events or routine clinical assessments) that they had not reported in publications.

Creating the database and contacting authors in this way would be an excellent way to try to reduce some aspects of publication bias (where outcomes that do not appear to be statistically significant are seen as less interesting and not published). It also meant that we could potentially collect enough data across studies to formally assess effects of omega- 3 , omega- 6 and total PUFA on the diagnosis of common diseases, such as 
diabetes, depression and cancers, even where few studies formally aimed to assess these outcomes. Within the database we included enough studies to develop a method for assessment of compliance, an important aspect of validity in dietary trials. Development of the database also provided the possibility to make the database accessible to others, to use as the basis of systematic reviews assessing additional outcomes to those requested by WHO or for future updates.

This paper discusses the production of the database of long-term RCTs assessing higher vs lower omega-3, omega- 6 and total PUFA intake on any outcome, presents that database (in supplementary file 1; supplementary file 2; supplementary file 3) for others to use and provides details of the methodology used in the set of reviews assessing health effects. Outcomes commissioned by WHO, considered key in their prioritisation process, were cardiovascular mortality, cardiovascular events, coronary heart disease, atrial fibrillation, lipids, stroke, all-cause mortality and adiposity (all published in three reviews ${ }^{1-3}$ ), type 2 diabetes, ${ }^{4}$ depression, ${ }^{5}$ neurocognition (including dementia), ${ }^{6}$ breast cancer ${ }^{7}$ and inflammatory bowel disease (IBD). ${ }^{8}$ We grouped the $27 \mathrm{WHO}$ reviews into eight papers, and all were pre-registered on the Cochrane Library ${ }^{1-3}$ or PROSPERO ${ }^{5-9}$ We have also used the database to assess effects on musculoskeletal and functional outcomes in older adults (a systematic review not requested by WHO). ${ }^{10}$

\section{OBJECTIVES}

The primary objective of this set of systematic reviews was to determine health effects of higher versus lower omega-6, omega-3 (alpha-linolenic acid (ALA) or longchain omega-3 (LCn3)) or total PUFAs intake using RCTs.

Secondary objectives included creation of a dataset of RCTs with a duration of $\geq 24$ weeks comparing higher with lower omega-3, omega- 6 or total PUFA, regardless of reported outcomes; and assessment of effects of higher omega-6, ALA, LCn3 and total PUFA on mortality; cardiovascular diseases (CVDs); IBD; cancers; glucose metabolism and diabetes; neurological outcomes, including cognition, depression and anxiety; musculoskeletal and functional outcomes; measures of body fatness; and serum lipids over appropriate durations.

\section{METHODS}

\section{Criteria for considering studies for this review}

We carried out this set of reviews in two stages. First, we created a database of all ongoing and completed RCTs of higher versus lower omega-6, ALA, LCn3 and total PUFA with a duration of $\geq 24$ weeks. From this database of relevant studies, we selected RCTs for individual reviews.

Six months was chosen as the minimum duration after considering equilibration of PUFAs into body tissues. Careful work by Browning et al ${ }^{11}$ suggests LCn3 supplements equivalent to one portion/week of oily fish reach
95\% of maximal incorporation by 5 days for eicosapentaenoic acid (EPA) in plasma phosphatidylcholine (95\% CI 0 to 18 days) but by 273 days for docosahexaenoic acid (DHA) into blood mononuclear cells (95\% CI 0 to 670 days). While this suggests individual variability, on average, all compartments except blood mononuclear cells had equilibrated by 117 days. The authors stated 'EPA and DHA reached a maximum in platelets in 3-4 weeks and 1-2 months, respectively, and in blood mononuclear cells in 6-9 months'. We chose 6 months (24 weeks) as the minimum duration of intervention to allow equilibration of most body compartments. For specific reviews with outcomes needing additional months for body composition changes to affect health, we set the minimum trial duration at 1 year.

\section{Inclusion criteria for the RCT database}

We included RCTs with a duration of $\geq 24$ weeks ( $\geq 168$ days) of continuous involvement and comparing higher with lower omega-6, omega-3 (ALA or LCn3) and/or total PUFA intakes. We included studies reported as full text, abstracts only, trials registry entries and/or unpublished data. We included cluster-randomised studies with $\geq 6$ clusters. Participants were adults ( $\geq 18$ years old), but we excluded studies of participants who were pregnant or acutely ill (with diagnosed current cancer, undergoing heart or renal transplantation, with HIV or AIDS, on haemodialysis, with IgA glomerulonephritis or any other renal problem except in diabetes). Our reasoning was to exclude people with conditions that may affect the relationship between polyunsaturated fats and cardiovascular events. Studies were included in the database regardless of reported outcomes.

Interventions could include dietary supplementation, a provided diet and/or advice on diet. Supplementation had to be in oil or capsule form, or as foodstuffs provided, to be consumed by mouth (we excluded enteral/parenteral feeds and enemas). Studies were included if they compared the effect of this intervention with usual diet; no advice; no supplementation or placebo (as appropriate); or with lower omega-3, omega- 6 and/or total PUFA intake, so long as they created higher versus lower omega-3, omega-6 or total PUFA arms. We also included trials directly comparing omega-3 with omega- 6 .

Omega-3 trials could aim to increase LCn3 and/ or ALA. LCn3 includes EPA, DHA and docosapentaenoic acid in oily fish, fish, concentrated or algal oils. ALA includes refined ALA or products such as oils with an ALA content of $\geq 10 \%$ of the total fat content, such as linseed (flax), canola (rapeseed), perilla, purslane, mustard seed, candlenut, stillingia or walnut as a food or oil, made into a spreading fat or supplementing another food. Sources and types of omega- 3 and omega- 6 fatty acids are discussed and defined in supplementary text 1 (supplementary file 4). Omega-6 included linoleic acid (LA), gamma-linoleic acid (GLA), arachidonic acid (AA) or any combination, as a supplement or dietary component high in omega-6, such as sunflower oil. Omega-6 
advice, foodstuffs or supplements had to aim to increase or decrease omega-6 intake or, if no clear aim was stated (but implied, such as aiming to provide a 'heart health' or a 'Mediterranean' diet), the intervention had to achieve an increase or a decrease of $\geq 10 \%$ of baseline omega- 6 . We included trials as increasing total PUFA intake where they stated an aim to increase total PUFA or a dietary component high in total PUFA intake, such as vegetable oil, or where the intervention led to an increase or a decrease of $\geq 10 \%$ of baseline total PUFA.

Studies with multiple risk factor intervention on lifestyle factors, such as weight, smoking, physical activity, or dietary interventions not involving dietary fats, were not included, except where the other intervention was a direct replacement for polyunsaturated fats or the effect of diet or supplementation could be separated from other interventions.

\section{Search methods for identification of studies}

We ran two sets of searches on CENTRAL (Cochrane), MEDLINE and EMBASE to 27 April 2017, and on ClinicalTrials.com and the WHO International Clinical Trials Registry Platform to September 2016. We also checked included trials of relevant systematic reviews and wrote to authors of included studies for additional studies and trial data (including unpublished outcome data). The first search strategy for MEDLINE (Ovid) was adapted from Al-Khudairy et $a l^{12}$ and was also used to locate trials of polyunsaturated fats (see supplementary text 2 in supplementary file 4 ). The second set of search strategies aimed to locate omega-3 trials and was adapted from Hooper et $\mathrm{ll}^{13}$ (see supplementary text 3 , supplementary file 4). These complex strategies were adapted for use in the other databases. The Cochrane sensitivity and precision-maximising RCT filter was applied to MEDLINE (Ovid) and for EMBASE, and terms recommended in the Cochrane Handbook for Systematic Reviews of Interventions were applied. ${ }^{14}$ To ensure the database is up to date, we re-searched all ongoing trials in December 2018 and included four previously ongoing trials, ${ }^{15-18}$ adding data on a further 50239 participants in trials from 1.0 to 7.4 years to dataset 1 (supplementary File 1).

Titles and abstracts located by these searches were downloaded, de-duplicated into EndNote software, assessed as a single set for inclusion in the database and used as the wider study pool from which included studies for individual reviews were selected. Included studies in all identified relevant systematic reviews were checked, and additional publications of included trials were obtained. Attempts were made to obtain full-text translations and/ or evaluations of all relevant non-English articles.

\section{Assessment of inclusion}

Review methods were based on those of the Cochrane Collaboration. ${ }^{19}$ Two review authors independently screened titles and abstracts for inclusion into the wider database (using EndNote software) and coded them as 'retrieve' (eligible or potentially eligible/unclear) or 'do not retrieve'. All those coded for retrieval by either reviewer were collected in full text, and two review authors independently screened the full text, assessed studies for inclusion, and identified and recorded reasons for exclusion of ineligible studies ( $\mathrm{LH}$ and one other reviewer) using an inclusion/exclusion form. We resolved disagreements through discussion or consulted a third reviewer (AA). We identified and excluded duplicates and collated multiple publications and abstracts for each trial (as studies rather than publications were the unit of interest). We recorded selection to allow completion of the Preferred Reporting Items for Systematic Reviews and Meta-Analyses flow diagram. The database was compiled within RevMan software ${ }^{20}$ in the format of Cochrane Characteristics of Included Studies tables (so additional training was not needed) and transcribed into Word, with references added in EndNote to create the Database.

\section{Inclusion criteria for specific reviews}

Database studies were assessed for inclusion into specific reviews. Trials of $\geq 12$ months' duration were included in CVD, mortality and cancer reviews, ${ }^{1-3} 7$ where they assessed any of the review's primary or secondary outcomes (table 1). Trials of $\geq 6$ months were included into the glucose metabolism and diabetes, depression and anxiety, IBD and inflammatory markers, and neurocognitive and functional outcomes reviews, ${ }^{4682} 22$ where they assessed any of the review's primary or secondary outcomes (table 2). For each study included in a review, all primary, secondary and tertiary outcomes were data extracted.

\section{Data collection and analysis for trials included in at least one systematic review}

A data extraction form was designed for this set of reviews and tested by each reviewer on a common 'training' study. Two review authors each extracted study characteristics independently in duplicate onto a Word data extraction form (by hand or electronically). Characteristics included bibliographical details, methods (study design, total duration of study, details of any 'run-in' period, number of study centres and location, study setting, withdrawals and date of study), details of participants, interventions, comparators and outcomes, process data (dietary intake or body fat data), trial funding, risk of bias assessments, trial registration data, effect modifiers and adverse events. Dichotomous data from dietary advice studies were extracted at the latest point available in the trial (regardless of the amount of reinforcement of the original dietary message), while dichotomous data from supplemental studies were extracted to the point that supplementation ended, or the trial ended, whichever was earlier. Continuous data were extracted at the latest point available in fixed-term trials, but in studies where participants were followed up for varying durations, participants' data were extracted from the first time point following the mean trial duration. Data from periods following the end of a trial were not used in the meta-analysis. Where papers 
Table 1 Primary, secondary and tertiary outcomes for the systematic reviews assessing effects of omega- 3 , omega- 6 and total polyunsaturated fatty acid on cardiovascular outcomes, cancers and mortality, including only trials of $\geq 12$ months duration

Primary and secondary outcomes-

studies were included whenever these

outcomes were assessed by trialists

(even if not fully or appropriately

reported). All these outcomes were data extracted and analysed when data were found.

\begin{tabular}{|c|c|c|}
\hline Review & found. & extracted and analysed. \\
\hline CVD and mortality ${ }^{1-3}$ & $\begin{array}{l}\text { Cardiovascular mortality. } \\
\text { Cardiovascular events. } \\
\text { Coronary heart disease events. } \\
\text { Lipids. } \\
\text { Stroke. } \\
\text { All-cause mortality. } \\
\text { Adiposity. } \\
\text { Atrial fibrillation. } \\
\text { Myocardial infarction. } \\
\text { Sudden cardiac death. } \\
\text { Peripheral vascular events. } \\
\text { Heart failure. } \\
\text { Revascularisation. } \\
\text { Angina. }\end{array}$ & $\begin{array}{l}\text { Blood pressure. } \\
\text { QoL. } \\
\text { Economic costs. } \\
\text { Serious adverse events. }\end{array}$ \\
\hline Cancer $^{7}$ & $\begin{array}{l}\text { Cancer diagnosis, recurrence and } \\
\text { mortality. } \\
\text { Breast cancer diagnosis and mortality. } \\
\text { Markers of risk, such as prostate- } \\
\text { specific antigen for prostate cancer and } \\
\text { breast density for breast cancer. }\end{array}$ & $\begin{array}{l}\text { QoL. } \\
\text { Body weight. } \\
\text { Adiposity measures. } \\
\text { Dropouts. }\end{array}$ \\
\hline
\end{tabular}

CVD, cardiovascular disease; QoL, quality of life.

reported continuous results as change from baseline, we used these data; otherwise, we used data at the latest point available (so long as the change in either arm from baseline was greater than the difference between arms at baseline, to exclude trials with very unbalanced baselines). We did not impute change data.

Disagreements were resolved by discussion and/or a third reviewer (LH or AA). For dichotomous outcomes, we extracted the number of participants in whom an outcome was assessed and the number of participants experiencing the outcome for each study arm. For continuous outcomes, the number of participants assessed, the mean and the variance in each arm were extracted (SDs were calculated from other variance data using the RevMan calculator).

Risk of bias was assessed using the Cochrane Risk of Bias tool ${ }^{23}$ and two additional domains, independently in duplicate alongside data extraction. We assessed attention bias and compliance as we felt these are important in dietary trials:

1. Random sequence generation (selection bias).

2. Allocation concealment (selection bias).

3. Blinding of participants and personnel (performance bias).

4. Blinding of outcome assessment (detection bias).

5. Incomplete outcome data (attrition bias).

6. Selective outcome reporting (reporting bias).
7. Attention bias (an aspect of performance bias, intervention participants receive more time and/or attention from study and/or health personnel during the trial).

8. Compliance (we considered studies to be at low risk of compliance bias when compliance was assessed, results of compliance assessment were clearly reported for all arms and where most participants appeared to have taken $\geq 75 \%$ of the intended fatty acid dose).

Further details about assessment of each domain are found in supplementary table 1 (supplementary file 4). Compliance data are difficult to interpret and assess in dietary trials, as compliance can be assessed in many ways. These include counting return of unused supplements or foods, participant collection of foods or supplements, intake self-report (including diaries) and/or changes in body measures of fatty acids (eg, fatty acids in plasma cholesteryl esters). For these reviews, we developed an assessment method for compliance that incorporated all these possible assessment methods (detailed in supplementary figure, supplementary file 4). For one review, where trial inclusion relied heavily on compliance evidence, we were stricter, insisting on evidence of differences in body markers of total PUFA or of total serum cholesterol, between intervention and control arms. ${ }^{2}$

We graded each potential source as high, low or unclear risk of bias and provided details from the study reports, 
Table 2 Primary, secondary and tertiary outcomes for the systematic reviews assessing effects of omega-3, omega- 6 and total polyunsaturated fatty acid on key health outcomes, including trials of $\geq 6$ months' duration

Primary and secondary outcomesstudies were included whenever these outcomes were assessed by trialists (even if not fully or appropriately reported). All these outcomes were were found.

\begin{tabular}{|c|c|c|}
\hline Review & were found. & analysed. \\
\hline Depression and anxiety ${ }^{21}$ & $\begin{array}{l}\text { Depression } \\
- \text { Incidence. } \\
- \text { Continuous measures. } \\
-50 \% \text { reduction (remission). } \\
\text { Anxiety } \\
- \text { Incidence. } \\
- \text { Continuous measures. } \\
-50 \% \text { reduction (remission). }\end{array}$ & $\begin{array}{l}\text { Social participation. } \\
\text { QoL. } \\
\text { Carer stress. } \\
\text { Healthcare and patient costs. } \\
\text { Adherence. } \\
\text { Fidelity. } \\
\text { Adverse events. } \\
\text { Withdrawal rates. } \\
\text { Withdrawals due to non-compliance, lack of efficacy } \\
\text { and/or side effects. } \\
\text { Psychosis, suicidality, suicide and self-harm. }\end{array}$ \\
\hline IBD and inflammatory markers ${ }^{8}$ & $\begin{array}{l}\text { Remission. } \\
\text { Relapse. } \\
\text { Severity scores. } \\
\text { Diagnosis of inflammatory bowel } \\
\text { disease (new cases). } \\
\text { Inflammatory markers, including } \\
\text { C reactive protein, interleukin 6, } \\
\text { erythrocyte sedimentation rate and } \\
\text { faecal calprotectin. }\end{array}$ & $\begin{array}{l}\text { Corticosteroid, immunosuppressant, } \\
\text { immunomodulatory use. } \\
\text { QoL. } \\
\text { Adiposity. } \\
\text { Other inflammatory markers, such as TNFalpha, } \\
\text { ICAM-1 VCAM-1. }\end{array}$ \\
\hline Neurocognitive outcomes ${ }^{6}$ & $\begin{array}{l}\text { Change in cognitive function or } \\
\text { score, such as Mini-Mental State } \\
\text { Examination (MMSE), Alzheimer's } \\
\text { Disease Assessment Scale Cognitive } \\
\text { Subscale (ADAS-Cog) and Clinical } \\
\text { Dementia Rating (CDR). } \\
\text { Change in memory. } \\
\text { New diagnosis of dementia or } \\
\text { cognitive impairment. }\end{array}$ & $\begin{array}{l}\text { QoL. } \\
\text { ADLs for example, Barthel score. } \\
\text { Adherence or compliance. } \\
\text { Safety. } \\
\text { Tolerability. } \\
\text { Economic costs. } \\
\text { Mortality. }\end{array}$ \\
\hline Diabetes $^{4}$ & $\begin{array}{l}\text { New diagnosis of diabetes. } \\
\text { Prediabetes diagnosis, for example, } \\
\text { impaired fasting glucose (IFG), } \\
\text { impaired glucose tolerance (IGT) and } \\
\text { impaired glucose regulation (IGR). } \\
\text { Changes in glucose, for example, } \\
\text { fasting or postprandial glucose or } \\
\text { glycated haemoglobin (HbA1c). } \\
\text { Change in insulin or insulin resistance. } \\
\text { Homeostatic Model Assessment of } \\
\text { Insulin Resistance (HOMA-IR). } \\
\text { Progression of diabetes, for example, } \\
\text { change in treatment strategy, newly } \\
\text { diagnosed retinopathy, neuropathy or } \\
\text { nephropathy. }\end{array}$ & $\begin{array}{l}\text { Change in serum triglycerides. } \\
\text { Change in serum total cholesterol. } \\
\text { Change in body mass index or other measures of } \\
\text { adiposity. } \\
\text { All-cause mortality. } \\
\text { Diabetic mortality. }\end{array}$ \\
\hline Musculoskeletal and functional ability ${ }^{22}$ & $\begin{array}{l}\text { Fracture incidence. } \\
\text { Bone mineral density or bone mass. } \\
\text { Bone turnover markers. } \\
\text { Sarcopenia or dynapenia incidence. } \\
\text { Skeletal muscle mass. } \\
\text { Measures of functional status, for } \\
\text { example, Barthel Index, ADLs, } \\
\text { mobility scores. } \\
\text { Strength or physical performance. }\end{array}$ & \\
\hline
\end{tabular}

ADL, activity of daily living; QoL, quality of life. 
unpublished data and/or author correspondence to justify the judgement. We used this to assess summary risk of bias for each trial.

\section{Summary risk of bias}

Supplement or capsule-type trials were considered to be at low summary risk of bias where randomisation, allocation concealment, blinding of participants, and personnel and blinding of outcome assessors were all judged low risk. All other trials were considered at moderate or high risk of bias. A dietary advice or all-food provided-type trial was judged at low summary risk of bias where randomisation, allocation concealment and blinding of outcome assessors were all judged adequate (all others were at moderate or high risk). We originally planned to assess summary risk of bias in the same way for all trials, but the WHO NUGAG expert group requested we assess summary risk of bias slightly differently in different types of trials. Research suggests that poorly concealed allocation is associated with $40 \%$ greater effect size, so randomisation and allocation concealment are core items for risk of bias assessment. ${ }^{24}$ Lack of blinding is associated with smaller levels of bias, ${ }^{25}$ especially where outcomes are objectively measured, ${ }^{26}$ as were most of those in our reviews, but small amounts of bias can be important where effect sizes are small.

\section{Measures of treatment effect}

Dichotomous data were analysed as risk ratios (RRs) with $95 \%$ CIs and continuous data as mean difference (MD) with $95 \%$ CIs. Where studies assessed effects using different but comparable scales, we standardised scales so that they presented with a consistent direction of effect (multiplying by -1 as needed) then combined using standardised MDs. Skewed data reported as medians were discussed, and medians were added to relevant forest plots to allow visual comparison of findings (but not pooled).

Where included studies had more than two intervention arms and separate control arms for each intervention arm, the relevant control arm was used for each intervention arm. Where there was a single control group, we combined intervention arms to compare with the single control group or used the single most relevant intervention group (to prevent participants appearing more than once in any meta-analysis). In factorial trials where only one intervention was relevant to our reviews, we used the relevant factorial analysis, but where we were interested in several interventions (cf AlphaOmega and Diet and Reinfarction Trial), ${ }^{27} 28$ we ensured the two interventions were never combined in the meta-analysis.

To minimise missing data, we attempted to contact authors of included and ongoing RCTs (including those identified only via trials registry entries or conference abstracts), to request data on review outcomes and key methods information. An RCT was included in a review where data on at least one review outcome had been measured (and for dichotomous outcomes, at least one person had experienced the outcome). Due to funding constraints, we were not able to contact all study authors.

\section{Data synthesis}

We conducted meta-analyses only when participants, comparisons and outcomes were similar enough to address a sensible question. Primary analyses assessed effects of LCn3, ALA, omega-6 and total PUFA on primary outcomes. Meta-analyses were carried out in RevMan $2014 .{ }^{20}$ Differences between lower and higher PUFA intake arms were combined across studies using RR or MDs in random-effects meta-analysis. We used a random-effects model because dietary interventions are more complex and heterogeneous than many medical treatments, but we used fixed-effects meta-analysis in sensitivity analyses (to check that our results were robust to differing assumptions). We also carried out sensitivity analyses to assess effects of methodological rigour, retaining only studies at low summary risk of bias, studies at low risk of compliance bias and larger trials (randomising $\geq 100$ participants).

We used $\mathrm{I}^{2}$ to measure heterogeneity between trials in each analysis. ${ }^{29}$ Substantial heterogeneity $\left(\mathrm{I}^{2}>50 \%\right)$ was reported, and we explored possible causes using prespecified subgroup analyses. We intended to assess reporting biases using a funnel plot only for forest plots with $\geq 10$ trials; however, we sometimes ran funnel plots on key outcomes with only eight or nine trials (never $<8$ ), to assess possible biases.

\section{Subgrouping}

We explored potential heterogeneity in the effects of omega-6, ALA, LCn3 or total PUFA on primary outcomes by performing prespecified subgroup analyses for all reviews:

1. Intervention type (dietary advice, supplementary capsules, supplemental foods or all foods provided).

2. Dose of LCn3, ALA, omega-6 and total PUFA (and dose response), where dose was the difference in dose between two arms:

a. $\mathrm{LCn} 3$ intake: $\leq 150 \mathrm{mg} / \mathrm{d}, \quad>150$ to $\leq 250 \mathrm{mg} / \mathrm{d}$,
$>250$ to $\leq 400 \mathrm{mg} / \mathrm{d},>0.4$ to $\leq 2.4 \mathrm{~g} / \mathrm{d},>2.5$ to $\leq 4.4 \mathrm{~g} / \mathrm{d}$,
$>4.4 \mathrm{~g} / \mathrm{d}$.
b. ALA intake: $<5 \mathrm{~g} / \mathrm{d}, \geq 5 \mathrm{~g} / \mathrm{d}$.
c. omega- 6 intake: $\leq 4 \% \mathrm{E},>4 \% \mathrm{E}$.
d. total PUFA: $<1 \% \mathrm{E}$, one to $<2 \% \mathrm{E}$, two to $<5 \% \mathrm{E}$,
$\quad \geq 5 \% \mathrm{E}$.
3. Trial duration: 6 to $<12$ months where included, 12 to $<24,24$ to $<48$ and $\geq 48$ months.

4. Replacement of saturated fatty acids (SFA), monounsaturated fatty acids (MUFA), carbohydrate, other PUFA, nil and unclear.

5. Baseline risk of outcome (categories varied by outcome type).

6. Medication use $(\geq 50 \%$ of control group on relevant medication vs $<50 \%$ on such medication-statins for CVD outcomes, antidiabetics for diabetes outcomes and antidepressants for depression outcomes). 
7. Baseline LCn3, ALA, omega-6 or total PUFA intake (as appropriate).

8. Change in the omega-3/omega- 6 ratio.

Some reviews included additional subgroups, including participants' sex, participants' age and omega- 6 type (LA or GLA). The test for subgroup interactions in Review Manager V.5 was used. ${ }^{20}$

\section{Interpretation}

Outcome data were interpreted as follows:

1. Is there an effect on this outcome? (options: 'increased risk', 'decreased risk' or 'little or no effect'). For dichotomous data, $\mathrm{RR}<0.92$ or $>1.08$ for the highest quality evidence (main analysis and sensitivity analyses) suggested increased or decreased risk (otherwise little or no effect). Presence or absence of an effect was not decided by statistical significance.

2. For continuous outcomes, the effect threshold was a change of $\geq 5 \%$ from baseline, except for measures where effects are clearly cumulative (such as body weight) when $\geq 2 \%$ change from baseline was considered a relevant effect size.

3. Quality of evidence was assessed using GRADE assessment $^{30}$ (Grading of Recommendations Assessment, Development and Evaluation) for key outcomes, and displayed in summary of findings tables. We used the five GRADE considerations (risk of bias, consistency of effect, imprecision, indirectness and publication bias) to assess quality of evidence as it related to the studies that contributed to the meta-analyses using GRADEpro GDT software. ${ }^{31}$ We justified decisions on downgrading using footnotes.

4. Quality judgements were interpreted (as suggested by Cochrane) into clear statements about the evidence base (high-quality evidence suggests effect/lack of effect, and moderate-quality evidence suggests a probable effect; for low-quality evidence, there may be an effect, and for very low-quality evidence, any effects are unclear as the evidence is of very low quality).

5 . Where there was a suggested effect, the effect size was described using number needed to treat (NNT) or absolute risk reduction (ARR).

Using this method, some small statistically significant findings were considered to have 'little or no effect', and larger non-statistically significant findings could suggest increased or decreased risk.

\section{Patient and public involvement}

We did not involve patients or the public in this research at any stage.

\section{RESULTS}

The electronic searches generated 37810 hits, de-duplicated to 19772 titles and abstracts. We assessed these plus 88 studies included in related systematic reviews (53 studies included in two previous reviews, ${ }^{12} 13$ references found in 226 related systematic reviews but not picked up by our searches) and 986 potentially relevant trials registry entries ( 519 from ClinicalTrials.gov and 467 from WHO International Clinical Trials Registry Platform). A total of 2155 full-text papers, conference abstracts and trials registry entries were collected and independently assessed for inclusion by two reviewers. Included studies were grouped into 363 RCTs comparing higher with lower omega-3, omega-6 and/or total PUFA intake of at least 6 months duration-the trials database (see figure 1 for the flow diagram). Additional publications of these RCTs were searched (85 further items located), and authors were contacted where possible.

These 363 trials constitute the database of omega-3, omega- 6 and total PUFA RCTs with a duration of at least 6 months. Of these 363 included RCTs, 216 RCTs (dataset 1 , supplementary file 1 ) were included in at least one of our reviews of health outcomes, and consisted of 720 papers, abstracts and theses, 96 trial registrations and 91 sets of author correspondence. ${ }^{1-46-8} 2122$ The dataset includes references to all the paperwork we were able to locate on each trial, to support complete data extraction on methods, baseline characteristics and outcomes. These 216 trials were data extracted and risk of bias was assessed in duplicate, and their characteristics, risk of bias assessments, trials registry details and references are detailed in dataset 1 (supplementary file 1). The trials include 183791 randomised participants, and 18 trials randomised at least 1000 participants. Seven were harder-to-find trials only published in the form of conference abstracts, and one was not published in English. Details of which trials were included in which reviews are found in supplementary table 2 (supplementary file 4 ).

Ninety-five RCTs were included in the database but were not included in any of our current reviews (dataset 2 , supplementary file 2), so these were not data extracted or risk of bias assessed in duplicate (including 146 papers and abstracts, 8 trial registrations and 25 sets of author correspondence). Brief characteristics, trials registry details and references of these 95 trials have been detailed in dataset 2 (Supplementary file 2).

Of these 311 completed trials (within datasets 1 and 2):

- Twenty-seven trials altered ALA intake.

- Two hundred twenty-one trials altered LCn3 intake.

- Sixteen trials altered omega-3 intake without specifying whether ALA or LCn3.

- Forty-one trials altered omega-6 fats.

- Fifty-nine trials altered total PUFA (see details trial by trial in supplementary table 3 (supplementary file 4)).

Several trials had interventions that fitted more than one of these groupings, so the numbers do not add to 311. Of these 116 sets of author correspondence, most provided data on at least one additional outcome, and most provided methodological data.

For the remaining 52 trials, we found protocols, baseline data reports and/or trial registrations but no published outcomes, so we classified them as ongoing (including 28 papers or abstracts, 55 trial registrations and 5 sets of author correspondence). Brief characteristics, trials 


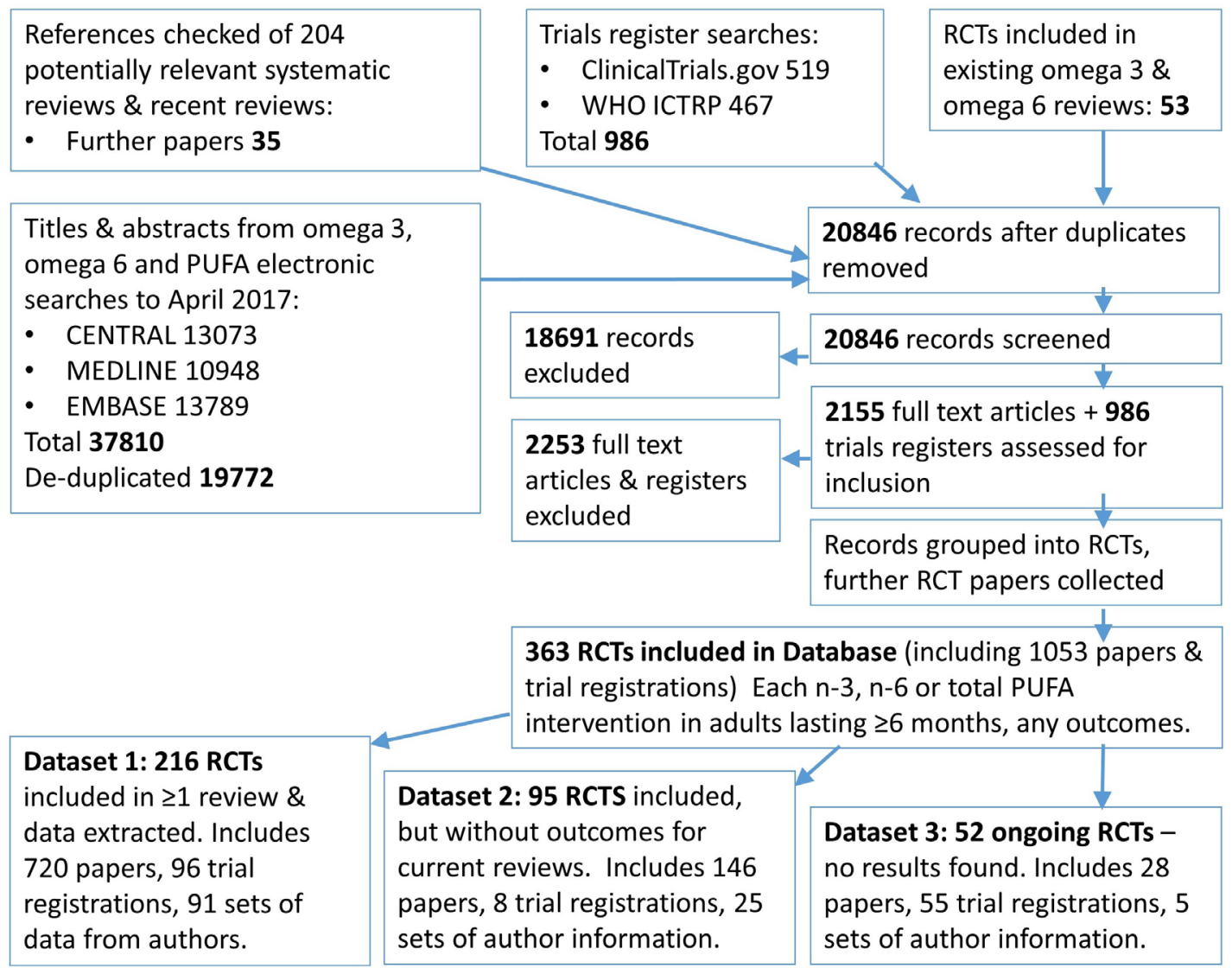

Figure 1 Preferred Reporting Items for Systematic Reviews and Meta-Analyses (PRISMA) flow diagram for the set of reviews. PUFA, polyunsaturated fatty acid; RCT, randomised controlled trial.

registry details and any references are detailed in Dataset 3 (supplementary file 3). Of these 52 RCTs, 5 were expected to be completed before 2012, and 8 had unclear completion dates but would have been expected to be published by 2015, given intervention duration and start dates, so 13 (25\%) appear to be outstanding or may constitute missing data.

Characteristics of the included trials and their risk of bias have been summarised review by review to enable readers to make sense of review findings. The tables of characteristics of included studies, along with references to the full set of relevant publications, and data on risk of bias constitute the database, including datasets 1, 2 and 3 . If researchers would like access to information provided by trialists, they are welcome to request it from the first author.

Risk of bias was formally assessed in duplicate for the 216 RCTs included in our set of systematic reviews. Of these, $42(19 \%)$ were found to be at low summary risk of bias (at low risk from selection and detection bias and at low risk of performance bias where trials provided supplements; figure 2 and supplementary file 1 ).

\section{DISCUSSION}

We have carefully compiled this database of longer term trials of omega- 3 , omega- 6 and total PUFA interventions, including completed and ongoing trials. This large set of trials has been partially characterised and assessed for risk of bias, and the full database is presented here as a background to our reviews, but also to allow others to use this database to conduct further systematic reviews more quickly and efficiently (without the need for extensive searching for these trials). We have aimed, where possible, to contact trial authors and to collect data on outcomes of interest to us but were not reported in formal publications. This has allowed us to access data not previously made available and has allowed some analyses (such as assessing effects of omega-3 fats on diabetes and depression diagnoses ${ }^{421}$ ) that were not previously informative.

Because high-quality systematic review methodology is important in understanding research evidence, we have aimed to be explicit in how we made decisions within this review. This allows discussion and disagreement and, we hope, will ultimately lead to improvements in methodology of nutritional systematic reviews. Some aspects of assessment of risk of bias within our reviews are worthy of note as they engendered a great deal of discussion between team members. When assessing blinding of participants and personnel (when assessing performance bias), we did not require that smell and taste were masked to achieve low-risk status, just that attempts had been made to create a visually equivalent placebo. As fish oil capsules can provide a fishy 

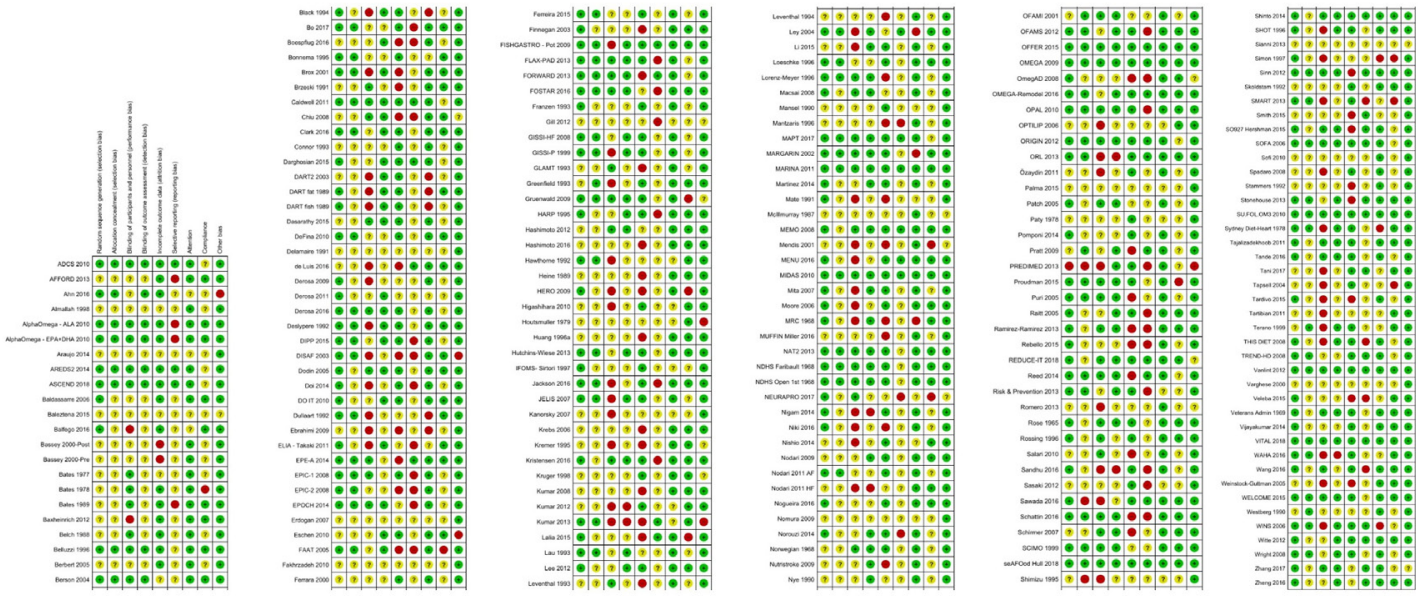

Figure 2 Risk of bias summary: review authors' judgements about each risk of bias item for each of the 216 included trials included in at least one of our systematic reviews (dataset 1, supplementary file 1).

after-taste, this may be a mistake, and some trials did carefully add citrus or mint flavours to intervention and control capsules, while others added a small amount of fish oil to control capsules to support masking. Almost no trials reported results of assessments to assess the success of masking, which would have been useful to support assessments.

When we established contact with trial authors and asked them questions about the methodology of their trial, we sometimes found that an author would say 'yes, allocation was concealed' or 'participants were not aware of whether they were taking the intervention or control' without providing requested information on methods to achieve them. We decided to take the author's word for the results of their methods in these cases, even where methodological justification was not provided. Finally, when checking whether all primary and secondary outcomes mentioned in the study protocol or trials register were also reported in study publications, we accepted studies as being at low summary risk of reporting bias when they reported additional outcomes (not mentioned earlier) in study reports (after some debate within our team). These boundaries were all much argued. We made allowances as these issues take trials to the limits of usual methodological reporting standards. However, we suggest that some of these issues may undermine trial methodological strength, even where trials were assessed by us as at low summary risk of bias.

We developed a system to assess risk of bias associated with compliance-compliance assessment is complicated in this database (and in many nutritional studies) as compliance could be reported in terms of returned capsules, self-reported intake (including diaries, recall and food frequency questionnaires), serum lipids and/or a variety of body composition measures. We developed a system to merge a variety of commonly reported measures (supplementary figure, supplementary file 4) and did not require plasma/ serum readings to accept compliance, except in the single review where compliance was used as an inclusion criteria. ${ }^{2}$ Assessment of compliance using different methods within a single trial was not always in agreement, so clearly, further research is needed on how best to assess compliance when including dietary trials in systematic reviews. We hope that our system will provide a starting point for further methodological work.

We developed and used the concept of a study at low summary risk of bias (a study at low risk from selection and performance bias, and also at low risk from performance bias where supplements were provided). We ran sensitivity analyses limiting to trials at low summary risk of bias. Where we had substantial numbers of trials (assessing cardiovascular effects of LCn3), we found that studies at low summary risk of bias appeared to suggest effect sizes closer to null than studies at moderate or high risk of bias. ${ }^{1}$ This suggests that this method of assessing summary risk of bias may have some utility.

This database has been used as the basis for our set of systematic reviews assessing health effects of omega-3, omega- 6 and total polyunsaturated fats ${ }^{1-46-821} 22$ and is available as a resource for others.

Acknowledgements We thank all of the authors of primary studies who kindly provided us with the best set of data available. These authors are acknowledged in the individual reviews in which their contributions have been used.

Collaborators The Polyunsaturated Fats and Health group includes Asmaa Abdelhamid (Norwich Medical School, University of East Anglia); Sarah Ajabnoor (Norwich Medical School, University of East Anglia); Faye Alabdulghafoor (Norwich Medical School, University of East Anglia); Lena Alkhudairy (Warwick Medical School, University of Warwick); Priti Biswas (Health Sciences, University of East Anglia); Julii Brainard (Norwich Medical School, University of East Anglia); Charlene Bridges (Cochrane Heart Group, University College London); Tracey J Brown (Norwich Medical School, University of East Anglia); Katherine Deane (Health Sciences, University of East Anglia); Daisy Donaldson (Norwich Medical School, University of East Anglia); Sarah Hanson (Health Sciences, University of East Anglia); Lee Hooper (Norwich Medical School, University of East Anglia); Oluseyi Florence Jimoh (Norwich Medical School, University of East Anglia); Nicole Martin (Cochrane Heart Group, University College London; Alex 0'Brien (Norwich Medical School, University of East Anglia; Karen Rees, Fujian Song (Norwich Medical School, University of East Anglia); Gabrielle Thorpe (Norwich Medical School, University of East Anglia); Xia Wang (Norwich Medical School, University of East Anglia); and Lauren Winstanley (Norwich Medical School, University of East Anglia).

Contributors LH conceived this database and set of reviews and obtained funding; LH and AA developed the core methodology for the reviews; LH drafted the searches, which were developed, refined, run and de-duplicated by the Cochrane Heart Group. All authors screened titles and abstracts, and assessed full-text papers for inclusion; LH and JSB searched trials registers and assessed entries for 
inclusion; LH and AA located full texts, and managed assessment and collection of titles, abstracts and full texts, data extraction and risk of bias assessment; all authors carried out data extraction and assessed risk of bias. LH, JSB and KHOD designed the risk of bias assessment; JSB developed assessment of compliance; FS advised on systematic review methodology; KHOD, AA and LH wrote to study authors; JSB, LH, KHOD and AA carried out data checks. All authors provided methodological support to other reviewers. LH, AA, JSB and KHOD entered data into RevMan and ran meta-analyses, and carried out sensitivity analyses and subgrouping. LH wrote the first draft of the paper. All authors critically read and commented on the final draft, and agreed to its submission. LH is the guarantor.

Funding The WHO Nutrition Guidance Expert Advisory Group (NUGAG) Subgroup on Diet and Health funded the research and was involved in its design, but not in data collection, analysis, interpretation or the decision to publish. The exception is that GRADE assessment was drafted by LH then discussed and agreed with NUGAG as part of guidance development. All researchers had full access to all the data (within a shared database) and take responsibility for the accuracy and integrity of the data.

Competing interests All authors had financial support via the University of East Anglia from the WHO for the submitted work; LH and AA were funded to attend WHO meetings and present review results.

Provenance and peer review Not commissioned; externally peer reviewed.

Data sharing statement All data relevant to the study are included in the article or uploaded as supplementary information. Our database of randomised controlled trials that assess effects of increasing omega- 3 , omega- 6 or total polyunsaturated fatty acids over at least 24 weeks is included in supplementary files to this BMJ Open paper. The database consists of three datasets, and for each included trial, we provide information on characteristics and risk of bias plus references to publications, trials registers and abstracts. Data are available on request; we hold some further data, such as our correspondence with authors. This will be made available to others on request to the corresponding author.

Open access This is an open access article distributed in accordance with the Creative Commons Attribution Non Commercial (CC BY-NC 4.0) license, which permits others to distribute, remix, adapt, build upon this work non-commercially, and license their derivative works on different terms, provided the original work is properly cited, appropriate credit is given, any changes made indicated, and the use is non-commercial. See: http://creativecommons.org/licenses/by-nc/4.0/.

\section{REFERENCES}

1. Abdelhamid AS, Brown TJ, Brainard JS, et al. Omega-3 fatty acids for the primary and secondary prevention of cardiovascular disease. Cochrane Database Syst Rev 2018;304. 11:CD003177.

2. Abdelhamid AS, Martin N, Bridges C, et al. Polyunsaturated fatty acids for the primary and secondary prevention of cardiovascular disease. Cochrane Database Syst Rev 2018;11:CD012345.

3. Hooper L, Al-Khudairy L, Abdelhamid AS, et al. Omega- 6 fats for the primary and secondary prevention of cardiovascular disease. Cochrane Database Syst Rev 2018;11:CD011094.

4. Brown TJ, Brainard JS, Song F, et al. Omega-3, omega-6 and total dietary polyunsaturated fat for prevention and treatment of type 2 diabetes mellitus: systematic review of randomised controlled trials. Br Med J 2018. submitted.

5. Hanson S, Biswas P, Jimoh O, et al. Dietary polyunsaturated fat for prevention and treatment of depression and anxiety. Prospero 2017:CRD42017056092.

6. Jimoh OF, Brainard J, Deane KA, et al. Dietary polyunsaturated fat for prevention and treatment of neurocognitive disorders. Prospero 2017:CRD42017019049.

7. Hanson S, Thorpe G, Winstanley L, et al. Effects of supplementary dietary polyunsaturated fat on cancer incidence. Prospero 2017:CRD42017056109.

8. Thorpe G, Ajabnoor S, Ahmed Z, et al. Dietary polyunsaturated fat for prevention and treatment of inflammatory bowel disease. Prospero 2017:CRD42017068704

9. Brown T, Song F, Wang X, et al. Dietary polyunsaturated fat for prevention and treatment of type 2 diabetes mellitus. Prospero 2017:CRD42017064110.
10. Abdelhamid A, Hooper L, Welch A. Polyunsaturated fatty acids for musculoskeletal health and functional status in older adults. Prospero 2017:CRD42017079211.

11. Browning LM, Walker CG, Mander AP, et al. Incorporation of eicosapentaenoic and docosahexaenoic acids into lipid pools when given as supplements providing doses equivalent to typical intakes of oily fish. Am J Clin Nutr 2012;96:748-58.

12. Al-Khudairy L, Hartley L, Clar C, et al. Omega 6 fatty acids for the primary prevention of cardiovascular disease. Cochrane Database Syst Rev 2015;11:CD011094.

13. Hooper L, Harrison RA, Summerbell CD, et al. Omega 3 fatty acids for prevention and treatment of cardiovascular disease. Cochrane Database Syst Rev 2004;20.

14. Lefebvre C, Manheimer E, Glanville J. Chapter 6: Searching for studies. In: Higgins JPT, Green S, eds. Cochrane Handbook for Systematic Reviews of Interventions: The Cochrane Collaboration, 2011. www.cochrane-handbook.org.

15. Bowman L, Mafham M, Wallendszus K, et al. Effects of $n-3$ Fatty Acid Supplements in Diabetes Mellitus. N Engl J Med 2018;379:1540-50.

16. Bhatt DL, Steg PG, Miller M, et al. Cardiovascular Risk Reduction with Icosapent Ethyl for Hypertriglyceridemia. $N$ Engl J Med 2019;380:11-22.

17. Hull MA, Sprange K, Hepburn T, et al. Eicosapentaenoic acid and aspirin, alone and in combination, for the prevention of colorectal adenomas (seAFOod Polyp Prevention trial): a multicentre, randomised, double-blind, placebo-controlled, $2 \times 2$ factorial trial. Lancet 2018;392:2583-94.

18. Manson JE, Cook NR, Lee IM, et al. Marine n-3 Fatty Acids and Prevention of Cardiovascular Disease and Cancer. N Engl J Med 2019;380:23-32.

19. Higgins JP, Green S. Cochrane Handbook for Systematic Reviews of Interventions Version 5.1.0 (updated March 2011): The Cochrane Collaboration, 2011.

20. Review Manager 5 (RevMan 5) [program]. Copenhagen: The Nordic Cochrane Centre: The Cochrane Collaboration, 2014.

21. Deane $\mathrm{KHO}$, Jimoh OF, Biswas $\mathrm{P}$, et al. Omega-3, omega-6 and total polyunsaturated fat for prevention and severity of depression and anxiety: a systematic review and meta-analysis of RCTs. 2018. submitted.

22. Abdelhamid AS, Hooper L, Sivakaran R, et al. Omega-3, omega-6 and total polyunsaturated fat for musculoskeletal health and functional status in older adults: a systematic review and metaanalysis of RCTs. 2018. submitted.

23. Higgins JPT, Altman DG, JACobotCSMG S, et al. Chapter 8: Assessing risk of bias in included studies. Higgins Jpt GS, ed. Cochrane handbook for systematic reviews of interventions Version 510: The Cochrane Collaboration, 2011. www.handbook.cochrane. org

24. Schulz KF, Chalmers I, Hayes RJ, et al. Empirical evidence of bias. Dimensions of methodological quality associated with estimates of treatment effects in controlled trials. JAMA 1995;273:408-12.

25. Savović J, Jones $\mathrm{H}$, Altman $\mathrm{D}$, et al. Influence of reported study design characteristics on intervention effect estimates from randomised controlled trials: combined analysis of metaepidemiological studies. Health Technol Assess 2012;16:1-82.

26. Wood L, Egger M, Gluud LL, et al. Empirical evidence of bias in treatment effect estimates in controlled trials with different interventions and outcomes: meta-epidemiological study. BMJ 2008;336:601-5.

27. Kromhout D, Giltay EJ, Geleijnse JM. n-3 fatty acids and cardiovascular events after myocardial infarction. $N$ Engl J Med 2010;363:2015-26.

28. Burr ML, Fehily AM, Gilbert JF, et al. Effects of changes in fat, fish, and fibre intakes on death and myocardial reinfarction: diet and reinfarction trial (DART). Lancet 1989;2:757-61.

29. Higgins JP, Thompson SG, Deeks JJ, et al. Measuring inconsistency in meta-analyses. BMJ 2003;327:557-60.

30. Atkins D, Best D, Briss PA, et al. Grading quality of evidence and strength of recommendations. BMJ 2004;328:1490-90.

31. GRADEpro GDT. GRADEpro GuidelineDevelopment Tool [program]: gradepro.org: McMaster University (developed by Evidence Prime, Inc), 2015. 